\title{
Sensors for the End-Cap Prototype of the Inner Tracker in the ATLAS Detector Upgrade
}

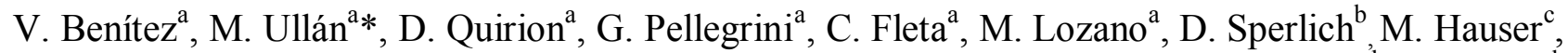
S. Wonsak ${ }^{\mathrm{c}, \mathrm{g}}$, U. Parzefall ${ }^{\mathrm{c}}$, K. Mahboubi ${ }^{\mathrm{c}}, \mathrm{S}$. Kuehn ${ }^{\mathrm{c}}$, R. Mori ${ }^{\mathrm{c}}$, K. Jakobs ${ }^{\mathrm{c}}$, J. Bernabeu ${ }^{\mathrm{d}}$, C. García ${ }^{\mathrm{d}}$, C. Lacasta ${ }^{d}$, R. Marco ${ }^{d}$, D. Rodriguez ${ }^{d}$, D. Santoyo ${ }^{d}$, C. Solaz ${ }^{d}$, U. Soldevila ${ }^{d}$ D. Ariza ${ }^{e}$, I. Bloch $^{\mathrm{e}}$,

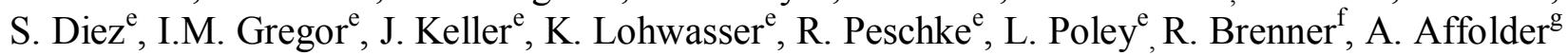

${ }^{a}$ Centro Nacional de Microelectrónica (IMB-CNM, CSIC), Barcelona, Spain

${ }^{\mathrm{b}}$ Humbold Universität Berlin, Germany

${ }^{c}$ Albert-Ludwigs-Universität Freiburg, Germany

${ }^{\mathrm{d}}$ IFIC-Valencia (CSIC, UVEG), Spain

${ }^{\mathrm{e}}$ DESY (Deutsches Elektronen-Synchrotron), Germany

${ }^{\mathrm{f}}$ Uppsala University, Sweden

${ }^{\mathrm{g}}$ University of Liverpool, United Kingdom

*Corresponding author. Centro Nacional de Microelectrónica (IMB-CNM, CSIC), Campus

Universitario de Bellaterra, 08193 Barcelona, Spain.

Phone: +34 9359477 00, Fax: +34 935801496.

E-mail: miguel.ullan@imb-cnm.csic.es

\begin{abstract}
The new silicon microstrip sensors of the End-cap part of the HL-LHC ATLAS Inner Tracker (ITk) present a number of challenges due to their complex design features such as the multiple different sensor shapes, the varying strip pitch, or the built-in stereo angle. In order to investigate these specific problems, the "petalet" prototype was defined as a small End-cap prototype. The sensors for the petalet prototype include several new layout and technological solutions to investigate the issues, they have been tested in detail by the collaboration. The sensor description and detailed test results are presented in this paper. New software tools have been developed for the automatic layout generation of the complex designs. The sensors have been fabricated, characterized and delivered to the institutes in the collaboration for their assembly on petalet prototypes. This paper describes the lessons learnt from the design and tests of the new solutions implemented on these sensors, which are being used for the full petal sensor development. This has resulted in the ITk strip community acquiring the necessary expertise to develop the full End-cap structure, the petal.
\end{abstract}

Keywords: Silicon radiation detectors; Strip sensors; HL-LHC; ATLAS Upgrade; Inner Tracker (ITk); End-cap.

\section{INTRODUCTION}

The LHC experiments at CERN will face a series of experimental upgrades in the following years: phase- 0 in 2014, phase-I in 2018, and phase-II around 2023. For phase-II, the accelerator team has lately announced the goal of achieving an ultimate luminosity of $7.5 \times 10^{34} \mathrm{~cm}^{-2} \mathrm{~s}^{-1}$ (a factor 7.5 with respect to the current nominal luminosity) as described in the Scoping Document [1]. The final goal is to reach a total integrated luminosity of $3000 \mathrm{fb}^{-1}$ by about 2035. Studies on detector design and technology are underway in order to adapt to the new conditions and requirements. The current ATLAS tracker, the so-called Inner Detector (ID), will be replaced by an all-silicon Inner Tracker (ITk). Highly integrated core structures called 'staves' in the barrel part and 'petals' in the forward region (End-caps), will be used to support the silicon strip detector modules. These modules, constructed by gluing the hybrid readout electronics directly onto the silicon sensors, are attached to the core structure. These structures are in research and development phase [2]. 
In the End-cap (EC), the sensors are identical on both sides of the petal and consist of six different geometries. A small stereo angle of $40 \mathrm{mrad}$ between strips is obtained with a $20 \mathrm{mrad}$ rotation of the sensor at each side of the petal. This permits the same sensor design to be used on both sides of the petal. The development of petal prototypes is an effort driven by a collaboration of institutes. The sensors in the EC will use the same technology as the barrel sensors [3]. The geometry of the EC is significantly more complicated, as it needs six rings with six different sensor types. An image of the petal structure can be seen in Fig. 1.

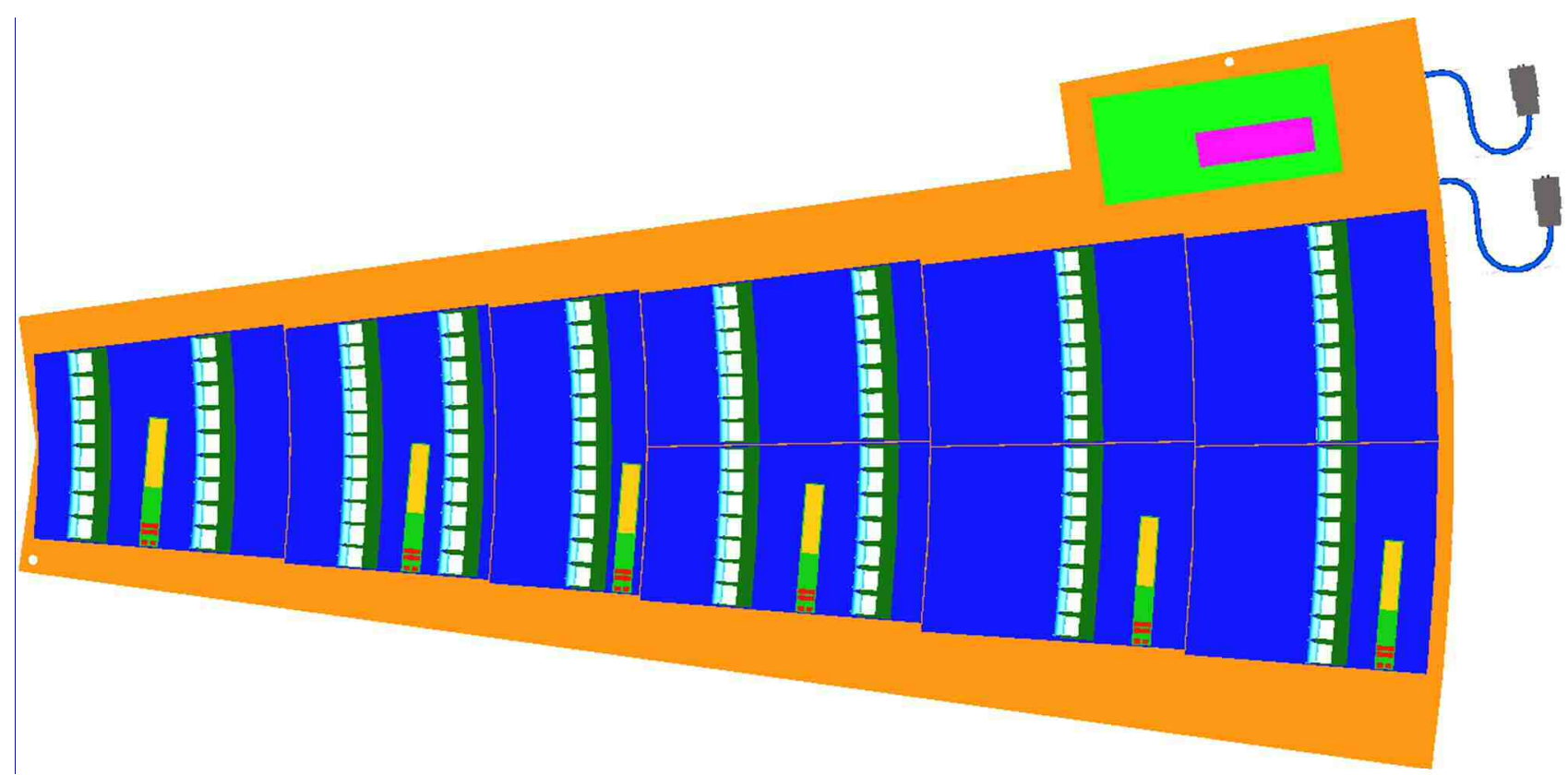

Figure 1: Petal structure proposed for the HL-LHC ATLAS Inner Tracker. The six different sensor types can be identified in dark color. In the three outer rings (to the right of the figure) the sensors are doubled, therefore there is a total of nine sensors in the petal with six different shapes.

\subsection{The petalet prototype}

The complex design of EC sensors is driven by the radial direction of the strips, the built-in stereoangle, and the trapezoidal shape of the sensors themselves. The combination of these conditions produce new challenges. In addition, the pitch varies strongly along the petal (from 67 to $106 \mu \mathrm{m}$ ), which leads to large bonding angles to the electronics if no pitch adapter is used.

The different sensors of the petal have varying shapes and dimensions at different radii. Each sensor type, due to the radial orientation of the strips and the built-in stereo angle, will have strips with different length, pitch and angle. Every ring will have a different number of channels, with the number increasing as they are placed further away from the beam. In the 3 outer rings, the area will be split in two identical sensors, given the size limitations of the commercially available 6" wafer process. More issues related to signal, commands and power access are expected in this region. In order to investigate the EC specific problems, a dedicated prototyping program with a small-scale petal (called the "petalet") was defined [4]. The petalet is a combination of a sensor representing the petal sensor for the innermost radius ("Big sensor") and two smaller sensors representing the area where the petal splits into two sensors ("Top sensors"). 
As far as the sensor design is concerned the most important goal of the petalet prototype is to address the different and particular problems associated with the petal sensor design, implementing them in a smaller-size prototype. The petalet also serves to prototype and test further components of a petal, such as the hybrids, bus tapes, support structures, cooling, readout of multiple modules and to perform assembly tests [5].

\section{SENSOR DESIGN}

Due to the complexity of the sensor structure, with built-in stereo-angle, different strip pitch, incomplete strips, embedded pitch adapters, etc., an automatic layout generation tool is required. In order to implement the construction algorithm, it was decided to use Python as the programming language. The automatic generation tool developed uses the Gdspy library [6] as base in order to create the final mask files [7].

There are three types of sensors on each side of the petalet: 'Big Sensor', 'Top Left' and 'Top Right', each of them has two strip rows which are designed separately using the automatic tool developed specifically for this project. The geometry and overall dimensions of the 3 types of petalet sensors can be seen in Fig. 2. The strips are designed with a $20 \mathrm{mrad}$ rotation with respect to their corresponding azimuthal angle, in order to obtain a $40 \mathrm{mrad}$ total built-in stereo angle between the front and back sensor strips when they are assembled on a petalet core. The strips are completely surrounded by a p-stop implant layer which is 8 microns wide, in mask, which is at 31 microns constant distance from the n-implant edge.

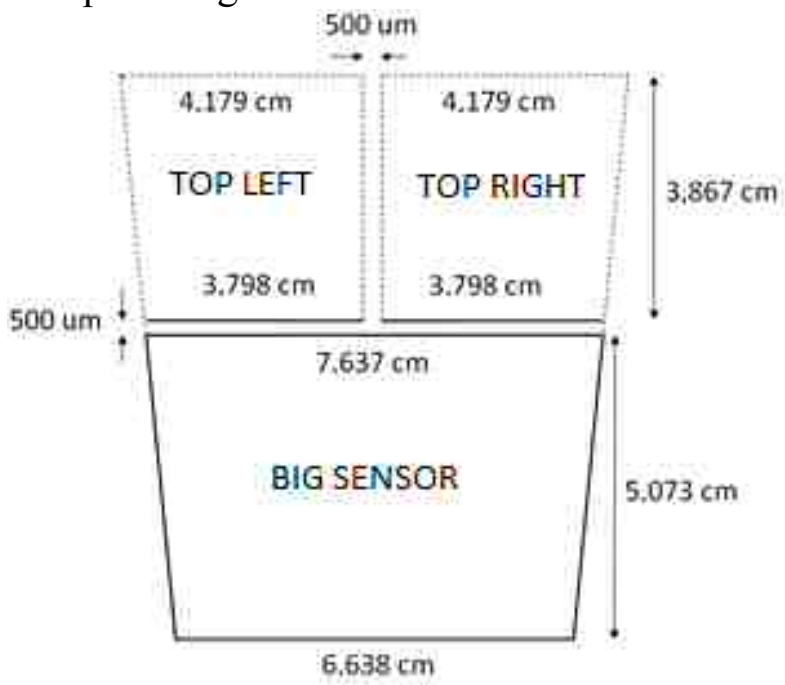

Figure 2: Geometry and dimensions of the three types sensors in the petalet.

\subsection{Incomplete strips}

The built-in stereo angle leads to incomplete strips at the sensor edges. These are strips that reach the sensor edge before they arrive to the bonding axis. For this reason they do not have a bond pad and cannot be readout by the electronics. For the first batches, in order not to lose active sensor area, incomplete strips were connected to their closest complete strip. When the added total length of incomplete strips was too long (three times higher than average strip length) the sensor was redesigned and a channel of the readout chip (ASIC) was reserved for those incomplete strips. The reserved channel will have a bonding pad placed as close as possible to the bonding axis, in consequence that pad must be placed on the incomplete strip closest to the last complete strip, and the remaining incomplete strips will be connected to it. 
For the final batches, AC-ganging [8,9] was implemented by creating a metal path between an incomplete strip and one complete strip that is not next to it (the third complete neighbor or further) in order to facilitate the identification of the signals coming from the incomplete strips. These two types of ganging incomplete strips can be can be observed in Fig. 3.
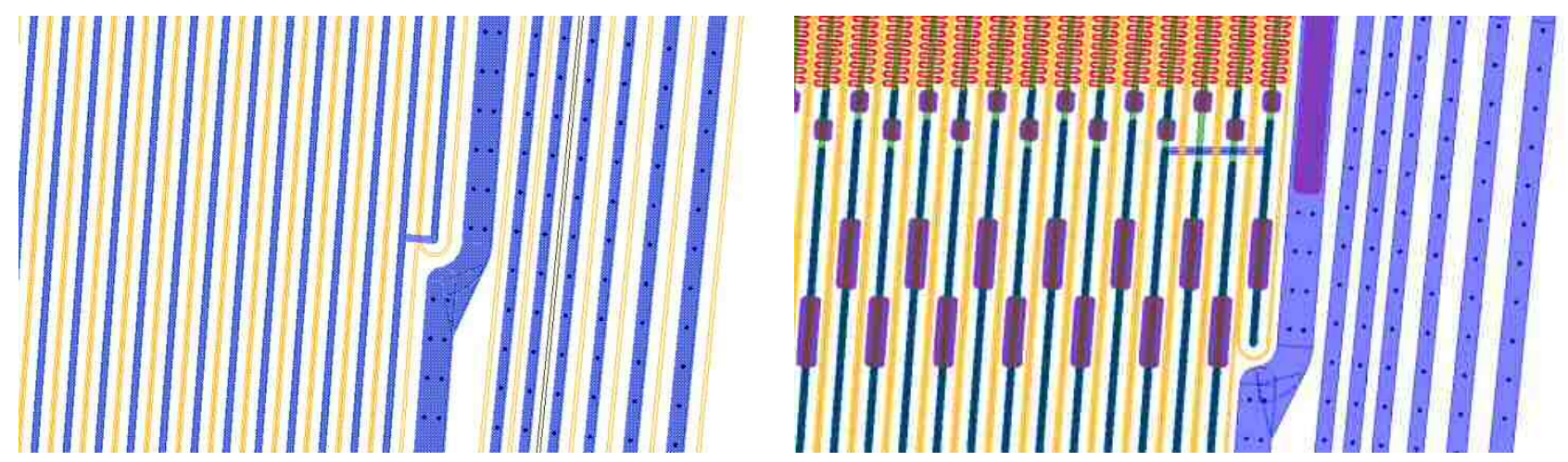

Figure 3: Detail of the two types of strip ganging for incomplete strips: to the closest complete strip (left) and so-called "AC-ganging" (right).

\subsection{Embedded pitch adapters}

The geometry of EC sensors resulted in bond pads which are not parallel and which have a pitch that differs from the pad pitch of the readout ASICs. This is not the best situation for automatic wirebonding machines; therefore, our solution is to build fan-in structures on the sensor, so-called embedded pitch adapters. The idea is to use a second metal layer to place an additional group of bonding pads on the sensor with a pitch that corresponds to that of the ASIC's bonding pads. These embedded pads are placed on the sensor and directly in front of the ASICs to facilitate the bonding. Second-metal tracks are then used to re-route the strip metal readout to these embedded pads. Fig. 4 illustrates the layout and how the embedded pads are connected to the strip readout. It can be also seen that a constant angle is selected in order to keep a constant pitch between second metal lines.

(a)

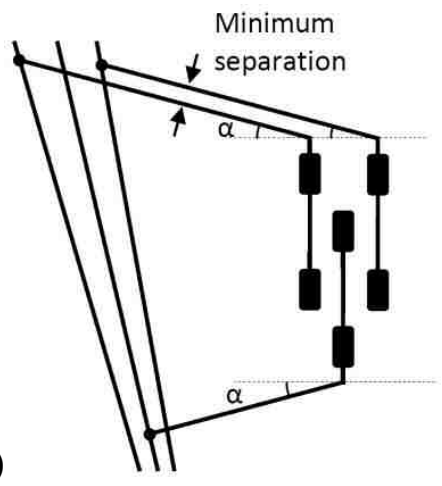

(b)

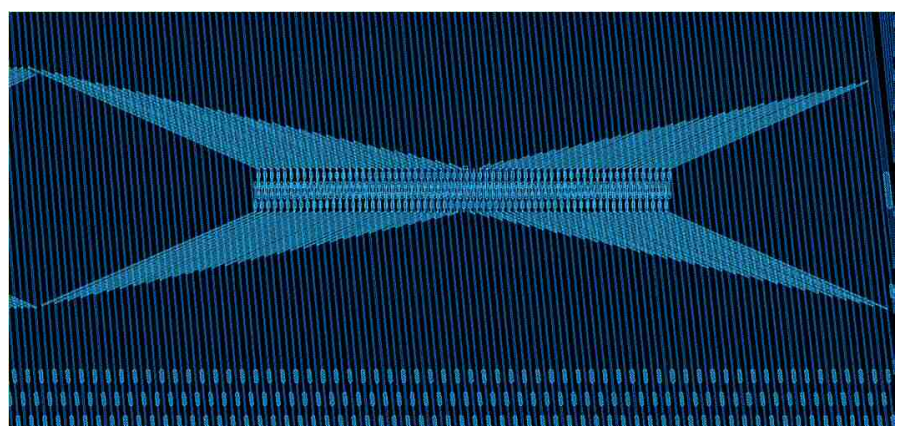

(c)

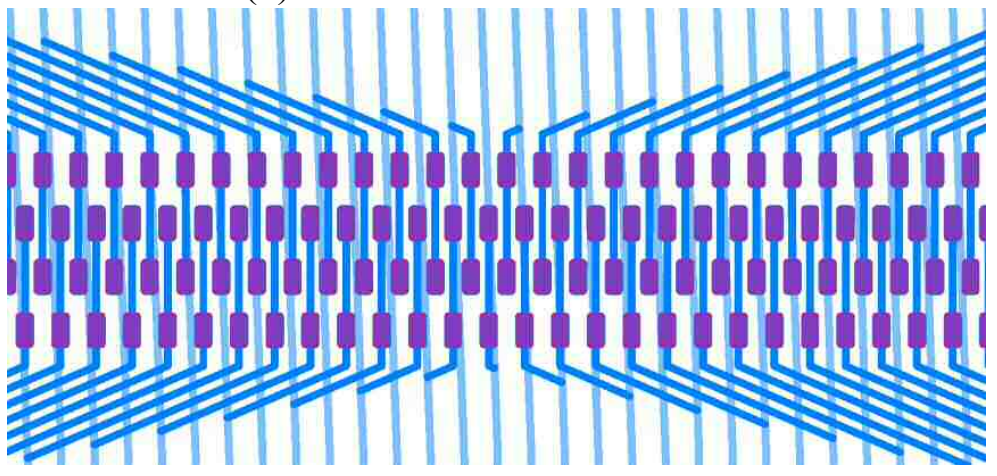


Figure 4: Design criteria of the embedded pitch adapters (a), their implementation (b), and detail of the structure (c).

The two main requirements when designing the pitch adapters were: minimum track length and maximum crossing angle. Both criteria try to minimize the effect of a second metal over the strips, in order not to degrade yield and to reduce cross talk and signal pick-up as much as possible. Embedded pads are placed along a horizontal line called embedded axis, which is parallel to the bonding axis. Embedded pads are placed in groups of 128 channels corresponding to the number of channels of the readout chips and according to the hybrid dimensions. The latter allows a choice between wirebonding to the sensor standard bonding pads or to the embedded pads. Both wire-bonding alternatives are separated by around $2 \mathrm{~mm}$ and electric circuit boards (hybrids) can be shifted down from their standard position to be bonded with the embedded pitch adapters. Initial tests on fabricated sensors show that the sensor tracking performance is not affected by the second metal layer [10]. Small increases in the interstrip capacitance have been observed, which lead to an increase of the readout noise and its variation from channel to channel. New designs have been fabricated, and are being tested [11], to try and reduce these effects.

\subsection{Full wafer design}

Final wafer layouts can be seen in Fig. 5. Other mini structures were also created with the design tool. Mini petal-like sensors with different strip pitch values around $100 \mu \mathrm{m}, 60 \mu \mathrm{m}$ and $45 \mu \mathrm{m}$, and mini barrel-like sensors, with dimensions of $1 \times 1 \mathrm{~cm}^{2}$, with one or two strip rows were designed. Test structures were also added around the wafer in order to obtain technological parameters.
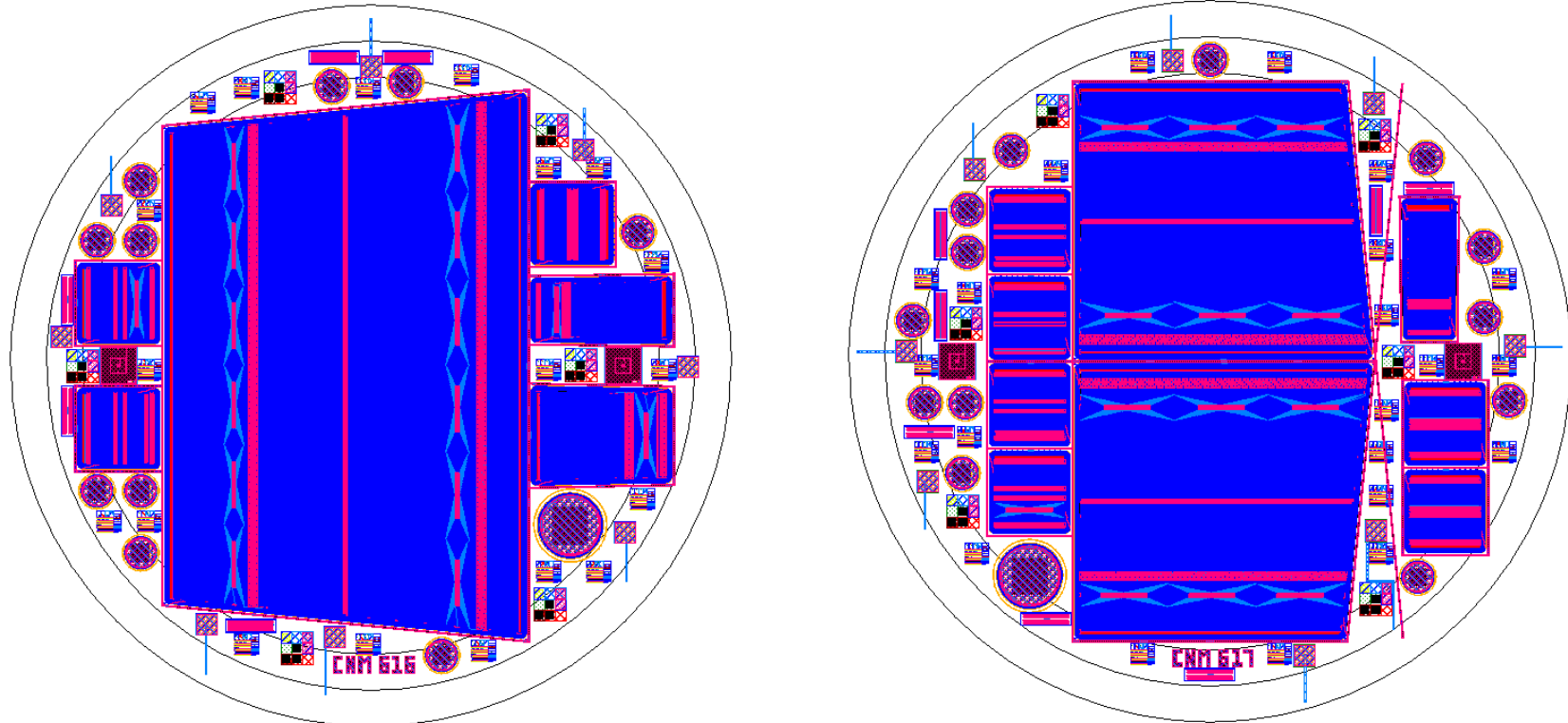

Figure 5: Final layout of the first mask set which contains a Big Sensor (left), and the second mask set, including both Top sensors (right).

\section{TECHNOLOGY AND FABRICATION}

The sensors for the petalet prototypes were fabricated in the clean room facilities at the Centro Nacional de Microelectrónica (IMB-CNM, CSIC), Barcelona, Spain. Float Zone (FZ), $285 \pm 15 \mu \mathrm{m}$ thick, 4-inches, silicon wafers, with (100) orientation, were used. High resistivity $(>10 \mathrm{k} \Omega \cdot \mathrm{cm})$, Boron-doped, p-type substrates have been chosen for the ITk sensors because they show better radiation hardness characteristics [12]. The p-type strip sensor manufacturing is a single sided process, which is also a major advantage compared to the radiation hard n-on-n option. The technology incorporates a p-stop implant for the proper inter-strip insulation. A total of seven 
photolithography steps were needed for the standard single-metal technology, and nine for the double-metal technology. Table 1 shows a summary of technological parameters of the sensors.

A total of 12 fabrication batches were completed in 21 months, resulting in 97 good quality sensors. 970 of them were distributed to the assembly institutes. Besides, 24 b-grade sensors and glass 10 dummies were distributed for assembly tests [5]. Fig. 6 shows Big and Top sensors after fabrication 11 and dicing. In order to perform mechanical and wire-bonding tests, dummy sensors were also fabricated. They are fabricated on $300 \mu \mathrm{m}$ thick glass substrates on which one metal layer is deposited to define the bonding pads.

\begin{tabular}{|l|c|}
\hline Wafer size & $150 \mathrm{~mm}$ \\
\hline Thickness & $285+/-15 \mu \mathrm{m}$ \\
\hline Orientation & $<100>$ \\
\hline Ingot & $\mathrm{FZ}$ \\
\hline Type & $\mathrm{P}$ \\
Substrate dopant & Boron \\
Resistivity & $>10 \mathrm{k} \Omega \cdot \mathrm{cm}$ \\
Strip implant (N) & $4.2 \mathrm{e} 15 \mathrm{~cm}^{-2}$ (Phosphorus) \\
p-stop implant & $4 \mathrm{e} 13 \mathrm{~cm}^{-2}$ (Boron) \\
Strip bias resistor & Polysilicon \\
Strip readout coupling & $\mathrm{AC}$ \\
Coupling oxide thickness & $37 / 100 \mathrm{~nm} *$ \\
Strip readout metal & $\mathrm{Al}(99.5 \%) / \mathrm{Cu}(0.5 \%)$ \\
\hline Metal thickness & $1.5 \mu \mathrm{m}$ \\
\hline
\end{tabular}

Table 1: Summary of technological parameters of the sensors.

* (The coupling oxide thickness has been modified during production as explained later)

\section{4. SENSOR MEASUREMENTS}

\section{4.1. Technological measurements}

21 After fabrication, electrical measurements on wafer level were performed in an automatic probe station, using the technological test structures implemented on the wafers. These test structures were used to obtain process parameters such as sheet resistance for conductive layers like $\mathrm{n}^{+}$implant,

24 polysilicon or metal, as well as contact resistance between layers, and capacitance between 
conductive layers. Later, these fabrication parameters are used as a reference to calculate and verify device parameters such as strip implant resistance, bias resistors, strip read-out resistance, or strip coupling capacitance. The polysilicon bias resistor mean obtained was $1.7 \mathrm{M} \Omega$, with a standard deviation of $0.3 \mathrm{M} \Omega . \mathrm{n}^{+}$implant sheet resistance was $21 \pm 1 \Omega / \mathrm{sq}$, which is equivalent to a strip resistance of $13.7 \pm 0.6 \mathrm{k} \Omega / \mathrm{cm}$. This value is in accordance with the collaboration specifications [3]. Regarding metal sheet resistance for the read-out metal a value of $26 \pm 0.2 \mathrm{~m} \Omega / \mathrm{sq}$ was obtained for single metal technology, and $60 \pm 0.2 \mathrm{~m} \Omega / \mathrm{sq}$ for double metal technology. These are equivalent to strip read-out resistances of $15 \pm 1 \Omega / \mathrm{cm}$ for single metal technology, and $30 \pm 1 \Omega / \mathrm{cm}$ for the first metal layer in sensors with embedded pitch adapters. The value for single metal technology meets specifications, while the one for the two metal layers is a bit higher than stated in the specifications. However, it should not be an issue for sensor operation. The second metal in the two metals technology shows a sheet resistance of $11 \pm 1 \Omega / \mathrm{cm}$. Capacitor test structures with area $800 \times 500 \mu \mathrm{m}^{2}$ (similar to the strip area) were tested in order to obtain reference values for the coupling capacitance. For the first batches, where the coupling capacitance oxide thickness was lower $(37 \mathrm{~nm})$, the capacitance of the test capacitor was $272.9 \pm 17 \mathrm{pF}$, while for the final batches where the oxide thickness was increased $(100 \mathrm{~nm})$, it was $156.0 \pm 10 \mathrm{pF}$. These values are equivalent to a capacitance per unit length in the strip of $136.4 \pm 8 \mathrm{pF} / \mathrm{cm}$ for the first batches with thinner oxides, and of $78.0 \pm 5 \mathrm{pF} / \mathrm{cm}$ for the final batches with thicker oxides, in good agreement with the strip tests as will be shown later. Both cases meet the specifications which require a value above $20 \mathrm{pF} / \mathrm{cm}$. As discussed in detail below, some yield issues were found in the coupling capacitors for the thin oxide cases. This is the reason why the oxide thickness was increased in the final batches.

\subsection{Sensor measurements}

For IV and CV measurements on the sensors, a manual probe station was used. The devices were placed in the measurement chamber at room temperature with a constant nitrogen flow in order to remove humidity and reduce surface currents. For IV characteristics, sensors were reverse biased from $0 \mathrm{~V}$ to $600 \mathrm{~V}$ with steps of $1 \mathrm{~V}$ and a settle time of 1 second. The current compliance level was set to $200 \mu \mathrm{A}$. Generally, reverse current levels are lower than $2 \mu \mathrm{A}$ when full depletion is reached. Fig. 7 (top) shows IV behavior for Big Sensors at room temperature. Typically no breakdown was observed before $300 \mathrm{~V}$, but an increase of current started from bias voltages of $250 \mathrm{~V}$. Leakage currents at a bias voltage of $200 \mathrm{~V}$ have a mean value of $0.6 \mu \mathrm{A} / \mathrm{cm}^{2}$ for the first batches and $0.2 \mu \mathrm{A} / \mathrm{cm}^{2}$ for the final batches, where some layout improvements where applied.

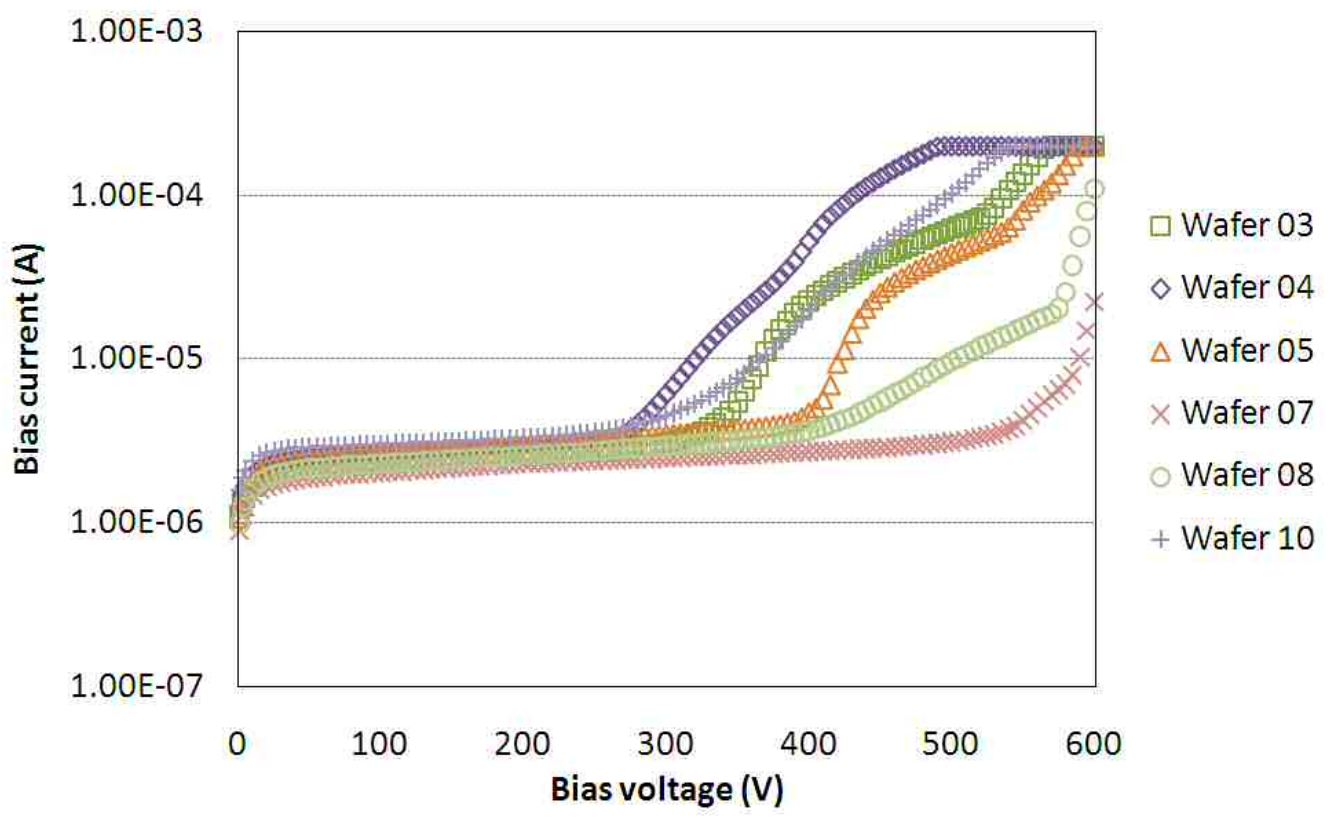




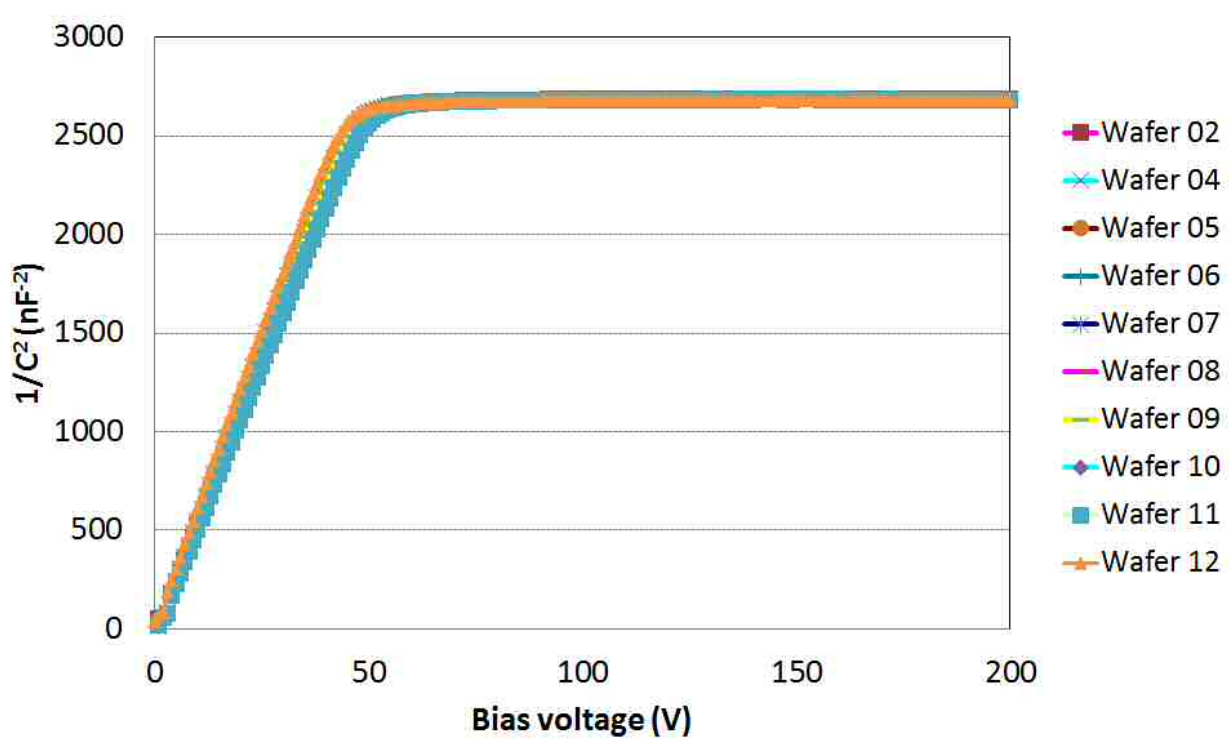

Figure 7: Results from IV measurements of the Big sensors (top), and the results from bulk capacitance measurements performed on $1 \times 1 \mathrm{~cm}^{2}$ pad sensors (bottom).

For the $\mathrm{CV}$ characteristics $1 \mathrm{x} 1 \mathrm{~cm}^{2}$ pad detectors fabricated on the wafers were used. The reverse voltage applied was swept from $0 \mathrm{~V}$ to $200 \mathrm{~V}$ (without additional probe for the bias ring), in $1 \mathrm{~V}$ steps. Capacitance meter terminals were connected between the bias pad and the sensor backplane metal, and the capacitance was obtained using the Cs-Rs model. Fig. 7 (bottom) shows the dependence of bulk capacitance with bias voltage. The measurements are made at $1 \mathrm{kHz}$ applied signal, as recommended by ATLAS12 specifications [3]. Sensors are fully depleted at $65 \pm 9 \mathrm{~V}$ for the initial batches and $47 \pm 4 \mathrm{~V}$ for the final batches, due to the use of slightly thinner and more resistive wafers. Both cases meet specifications, demonstrating a successful production.

\subsection{Single-channel measurements}

Since this was a prototype fabrication, not all sensor strips were measured systematically. Only some strips of a few sensors were sampled in order to obtain the qualitative behavior of all sensor parameters.

Coupling capacitance measurements were performed using two probes in one strip. One probe connected to the strip DC pad and used as ground, the second probe connected to the strip AC pad in order to apply the capacimeter signal. As required by the specifications, $1 \mathrm{kHz}$ signal was used in $\mathrm{Cp}$-Rp mode. The technological measurements mentioned above already predicted values according to specifications for coupling capacitance. Fig. 8 shows channel scans for two different sensors, where it can be seen that the values are above $20 \mathrm{pF} / \mathrm{cm}$, as required by the ATLAS12 specifications. In some of the sensors supplied to the collaboration for assembly, a high number of readout channels showing low gain were observed. This has been correlated with shorts in the coupling capacitance of the respective strips, associated with pinholes in the oxide. Nevertheless, the sensors with high number of bad channels are due to problems of a single wafer during fabrication. This event is very scarce (only two or three sensors have been found) and was not detected during sensor evaluation as a full QA inspection of all channels in all sensors has not been performed as explained before. In the final batches the oxide thickness was increased in order to overcome such problems. One of the sensors represented in Fig. 8 shows a higher coupling capacitance with a few bad strips probably related to "weak" pinholes in the oxide. This corresponds to the first batches where the coupling 
capacitor was thinner. The other sensor shows a lower coupling capacitance value, corresponding to a thicker oxide thickness, and no pinholes are seen.

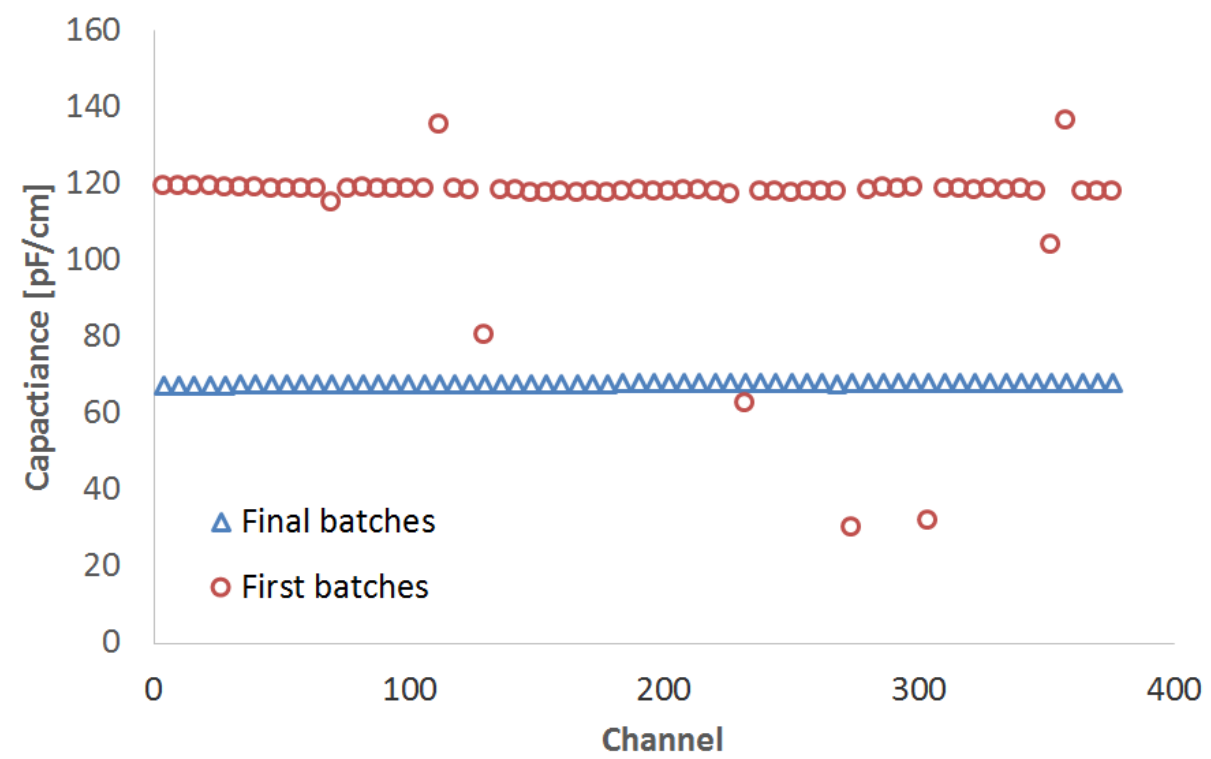

Figure 8: Strip coupling capacitance values obtained on different sensors by channel scans.

For interstrip capacitance measurements, the three probes method was used connecting them to the AC pads. The tested strip was connected to the capacimeter and its immediate neighbours were connected to ground. The sensor was fully depleted using $300 \mathrm{~V}$ reverse bias voltage and a $100 \mathrm{kHz}$ signal was applied in Cp-Rp mode. Fig. 9 (top) illustrates channel scans on different sensors. Very stable behavior is obtained. The measured values meet specifications, as the value is required to be lower than $0.8 \mathrm{pF} / \mathrm{cm}$ while the test results show a quite constant value of $0.5 \mathrm{pF} / \mathrm{cm}$.

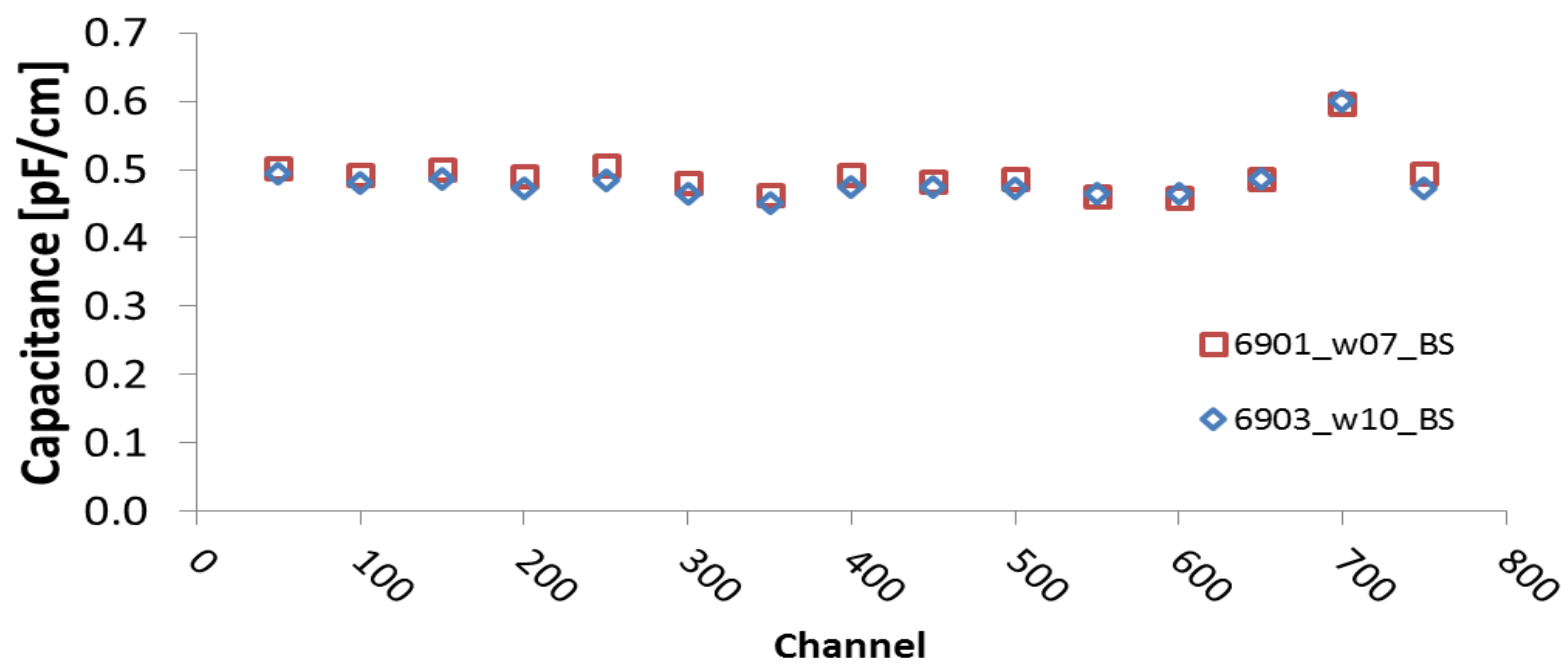




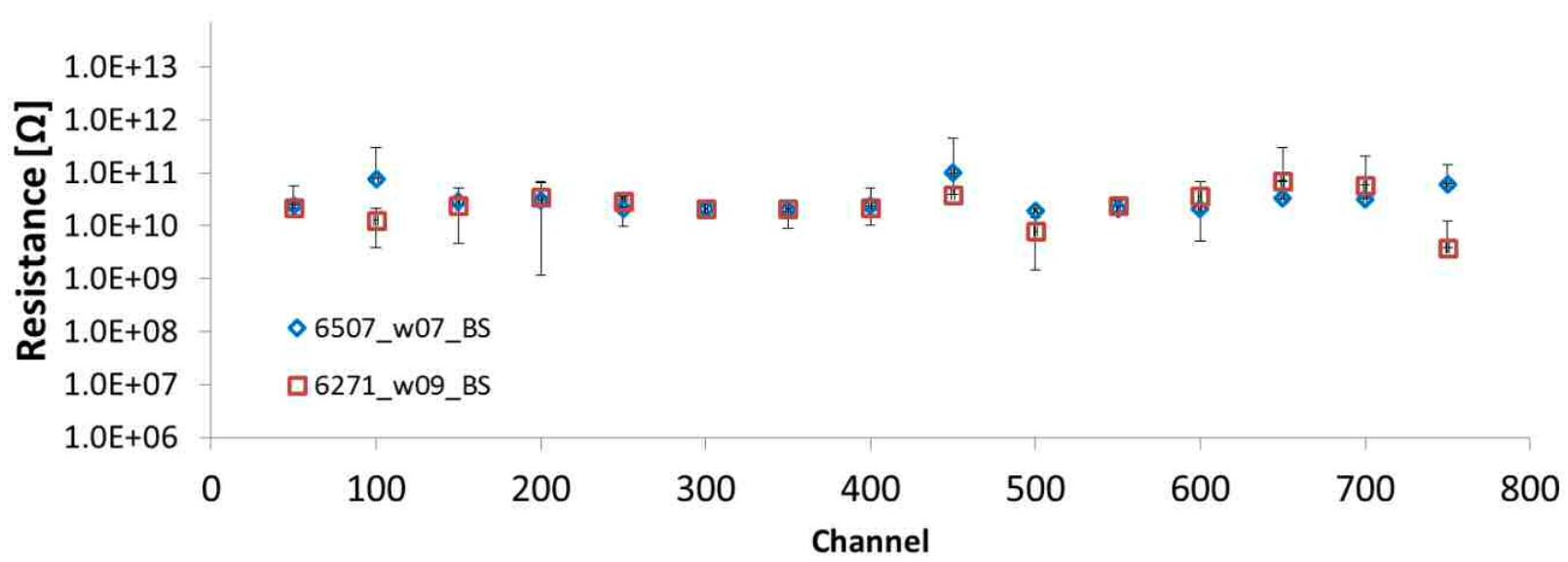

Figure 9: Interstrip capacitance (top) and interstrip resistance (bottom) values obtained on different sensors after channel scans.

Interstrip resistance measurements were done using three probes connecting them to the DC pads. The sensor is reverse biased at $300 \mathrm{~V}$. A semiconductor analyzer is used to set a voltage sweep from $-1 \mathrm{~V}$ to $1 \mathrm{~V}$ in $0.1 \mathrm{~V}$ steps to the tested strip on its DC pad, while the DC pads of the immediate neighbours are connected to ground. The current in the neighbour strips is measured and the interstrip resistance is obtained. Fig. 9 (bottom) shows channel scans on two different sensors and its variance along the strip row. It can be seen that the error bars are non-negligible due to the very low currents on the neighbour strips induced by the small voltage sweep. All values meet specifications as the obtained interstrip resistance is higher than ten times the bias resistance and the lower values are in the $\mathrm{G} \Omega$ order.

Further measurements were performed, such as leakage current stability, implant resistance, readout metal resistance, etc. Table 2 shows a summary of the main measurements performed.

\begin{tabular}{|c|c|c|}
\hline Measurement & ATLAS12 specs & Petalet sensors \\
\hline $\mathrm{CV}$ & $\mathrm{V}_{\mathrm{FD}}<300 \mathrm{~V}$ & $\mathrm{~V}_{\mathrm{FD}}<80 \mathrm{~V}$ \\
$\mathrm{IV}$ & $\mathrm{I}<2 \mathrm{uA} / \mathrm{cm}^{2} @ 600 \mathrm{~V}$ & $\mathrm{I}<2 \mathrm{uA} / \mathrm{cm}^{2} @ 200 \mathrm{~V}$ \\
$\mathrm{I}_{\text {leakage }}$ Stability & $<3 \% @ 600 \mathrm{~V} / 24 \mathrm{~h}$ & $<2 \% @ 120 \mathrm{~V} / 12 \mathrm{~h}$ \\
Ccoupling & $>20 \mathrm{pF} / \mathrm{cm}$ & $>40 \mathrm{pF} / \mathrm{cm}$ \\
Cint & $<0.8 \mathrm{pF} / \mathrm{cm} @ 300 \mathrm{~V}$ & $<0.7 \mathrm{pF} / \mathrm{cm} @ 300 \mathrm{~V}$ \\
Rint & $>10 \mathrm{Rbias} @ 300 \mathrm{~V}$ & $>1 \mathrm{G} \Omega @ 300 \mathrm{~V}$ \\
Rbias & $1.5 \mathrm{M} \Omega \pm 0.5 \mathrm{M} \Omega$ & $1.7 \mathrm{M} \Omega \pm 0.3 \mathrm{M} \Omega$ \\
Rimplant & $<20 \mathrm{k} \Omega / \mathrm{cm}$ & $13.7 \mathrm{~K} \Omega / \mathrm{cm}$ \\
Rmetal & $<15 \Omega / \mathrm{cm}$ & $15 \Omega / \mathrm{cm}(1 \mathrm{Metal})$ \\
\hline
\end{tabular}

Table 2: Summary of sensor characteristics and comparison with ATLAS12 specifications.

\subsection{Charge Collection Efficiency (CCE) measurements}

Several mini strip sensors have been tested with a fast analogue readout to obtain their charge collection efficiency. A ${ }^{90} \mathrm{Sr}$ beta source setup based on the ALIBAVA readout system was used [13]. Minimum ionizing particles generated signals in the sensors and their readout was triggered using two scintillators in coincidence. The measurements of four mini sensors of $1 \mathrm{x} 1 \mathrm{~cm}^{2}$ size with $\sim 100$ strips were performed at room temperature and relative humidity around $5 \%$. The results, the collected charge as a function of applied bias voltage, are given in Fig. 10. Three of the sensors had standard pads while the fourth sensor had embedded pads. All four sensors collect the expected charge of about 24000 electrons and show a good and comparable performance. 


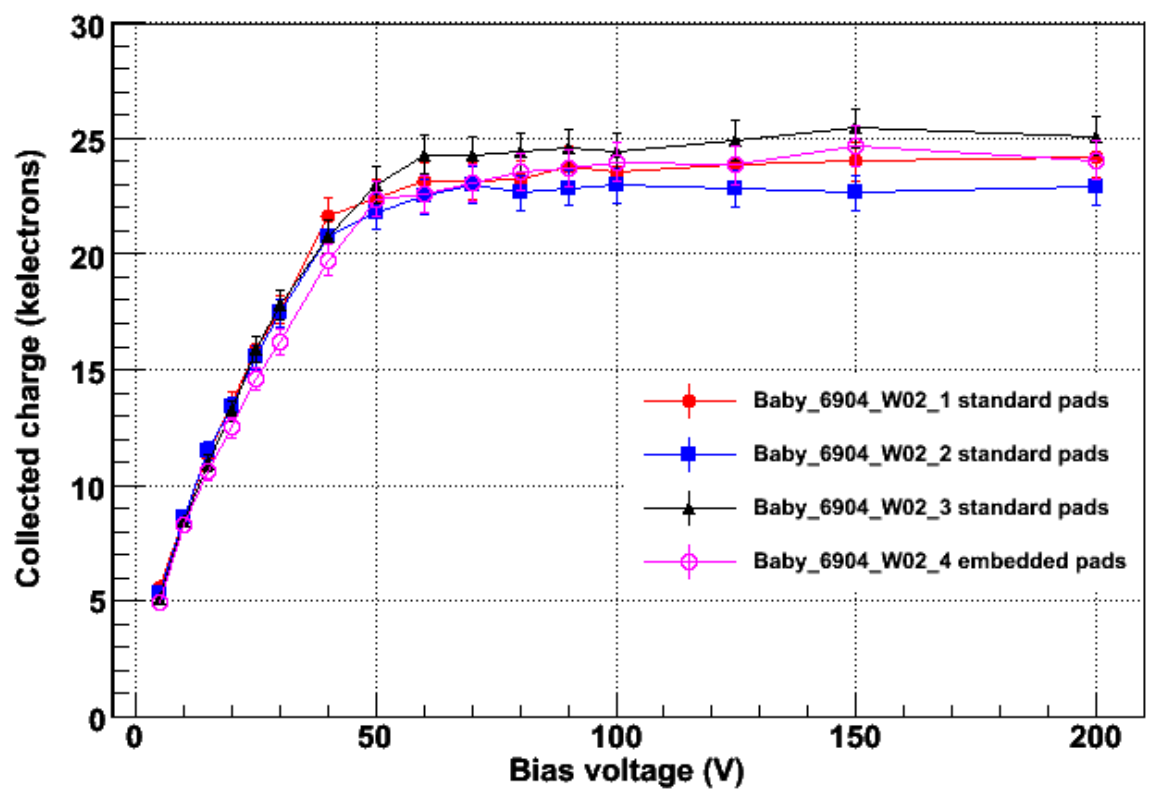

Figure 10: Collected charge of four miniature sensors $(1 \mathrm{x} 1 \mathrm{~cm} 2)$ measured, obtained with fast analogue readout. Sensors 1,2 and 3 have standard pads, sensor 4 has embedded pads.

\section{6}

\section{CONCLUSION}

The sensors for the prototypes of the End-cap of the Inner Tracker in the ATLAS Upgrade have been designed using newly developed automatic layout generation software. Novel layout and technological solutions have been implemented to solve the specific challenges of the ITk End-cap system, like ganging of incomplete strips for larger tracking area coverage, or embedded pitch adapters.

The sensors have been fabricated in the clean room facilities of the Centro Nacional de Microelectrónica (IMB-CNM, CSIC) in Barcelona, Spain. The tests show characteristics according to specifications under operating conditions. The layout and technology have been improved over the production process, and many lessons have been learnt in terms of the different new solutions implemented like the ganging of the incomplete strips and the embedded pitch adapters. More than 70 sensors have been delivered to the End-cap collaboration Institutes, which have been assembled into several petalet prototypes. In order to build the petalet modules, a fast readout flex board ("hybrid") is glued on top of the sensor. The hybrid contains the ASICs used for the readout of the channels. 24 ABCN25 ASICs [14] are used per petalet side. About 40 of such modules have been assembled using the well-performing sensors of the production. Measurements of leakage current were performed on the sensors after assembly and resulted in similar values compared to bare sensors testing, demonstrating the very good performance of the sensors and the reliability of the module building process. The use of these sensors has been fundamental for the development of the assembly tools and the expertise required to build the petalet prototype. At present the groups are in the process of developing the full End-cap structure, the petal, making use of all the expertise gained with the petalet program. In particular, the petal sensor development has strongly benefited from the petalet sensor development and tests.

\section{ACKNOWLEDGEMENT}


This work is supported and financed by the Spanish Ministry of Economy and Competitiveness through the Particle Physics National Program (ref. FPA2009-13234-C04-04) and co-financed with FEDER funds and by the European Social Fund and by the Ministry Of Science, Research and the Arts Baden-Wuerttemberg. This work is also financed by the Helmholtz Association, Germany. D.Q. acknowledges JAE-Doc funding.

\section{REFERENCES}

[1] The ATLAS Collaboration, ATLAS Phase-II Upgrade Scoping Document, CERN-LHCC-2015020, LHCC-G-166 (2015).

[2] S. Diez, et al. "A double-sided, shield-less stave prototype for the ATLAS Upgrade strip tracker for the High Luminosity LHC". Journal of Instrumentation, Volume 9, Issue 2, (2014).

[3] Y. Unno, et al. "Development of n+-in-p large-area silicon microstrip sensors for very high radiation environments - ATLAS12 design and initial results". Nuclear Instruments and Methods in Physics Research A 765 (2014), pp. 80-90

[4] C. Lacasta. "A proposal for a Petalet", in: Petals (Electrical) Strip Modules (Forward) and Sensor Layout, AUW, Nov. 2010.

[5] M. Aliev, et al. "A forward silicon strip system for the ATLAS HL-LHC upgrade". Nuclear Instruments and Methods in Physics Research Section A 730, (2013), pp 210-214.

[6] L. Gabrielli, Gdspy 0.2.7, http://gdspy.sourceforge.net/, Apr. 2011.

[7] V. Benitez, et al. "Design and Fabrication of Sensor Prototypes for the End-Cap Tracker of the ATLAS Upgrade”. 2012 IEEE Nuclear Science Symposium and Medical Imaging Conference Record (NSS/MIC) N14-158, pp. 1226-1230.

[8] Y. Unno, et al. "Development of $\mathrm{n}^{+}$-in-p large-area silicon microstrip sensors for very high radiation environments - ATLAS12 design and initial results", Nuclear Instruments and Methods in Physics Research Section A 765 (2014) pp 80-90

[9] R. Mori, et al. "Evaluation of the performance of irradiated silicon strip sensors for the forward detector of the ATLAS Inner Tracker Upgrade". Nuclear Instruments and Methods in Physics Research A. DOI: 10.1016/j.nima.2016.04.044 (Available on-line: http://dx.doi.org/10.1016/j.nima.2016.04.044).

[10]M. Ullan, et al. "Embedded pitch adapters for the ATLAS Tracker Upgrade". Nuclear Instruments and Methods in Physics Research A 732 (2013) pp. 178-181.

[11]M. Ullan, et al. "Embedded Pitch Adapters: a High-Yield Interconnection Solution for Strip Sensors". Nuclear Instruments and Methods in Physics Research A. DOI: 10.1016/j.nima.2016.04.019 (Available on-line: http://dx.doi.org/10.1016/j.nima.2016.04.019).

[12] Y. Unno, et al. "Development of n-on-p silicon sensors for very high radiation environments". Nuclear Instruments and Methods in Physics Research A 636 (2011), pp. S24-S30.

[13]R. Marco-Hernandez, "A portable readout system for microstrip silicon sensors (ALIBAVA)". IEEE Transactions on Nuclear Science NS-56 (2009) 1642.

[14]J. Kaplon, et al. "The ABCN front-end chip for ATLAS inner detector upgrade". Proceedings of the Topical Workshop on Electronics for Particle Physics (TWEPP 2008), Naxos, Greece. Sep 2008. 